\title{
Martial Arts as a Mindfulness in Motion: A Neurocognitive View
}

\author{
Bruna Brandão Velasques $^{1 *}$, Guaraci Ken Tanaka ${ }^{1}$, Renata Castro ${ }^{2}$, Adalgiza Mafra Moreno ${ }^{2}$, Alair Pedro Ribeiro ${ }^{1}$, \\ Silmar Teixeira ${ }^{3}$, Victor Hugo Bastos ${ }^{3}$, Marcos RG de Freitas ${ }^{4}$, Acary Bulle Oliveira ${ }^{5}$ and Marco Orsini ${ }^{1,3}$ \\ ${ }^{1}$ Rio de Janeiro Federal University, Brazil
}

${ }^{2}$ Nova Iguaçu University, Brazil

${ }^{3}$ Piaui Federal University, Brazil

${ }^{4}$ Medicine Department, Rio de Janeiro Federal University, Brazil

${ }^{5}$ São Paulo Federal University, Brazil

*Corresponding author: Bruna Brandão Velasques, Rio de Janeiro Federal University-IPUB-Institute,Rio deJaneiro, Brazil

\section{Introduction}

Mindfulness programs have given high visibility to bring the impact of the practice through a well-structured protocol that can be replicated over the years offering many clinical benefits as well as a greater understanding of neurophysiological mechanisms from immediate and long-term practice [1-5]. Since then, several protocols have been created to give specificity to the public suffering from mental health problems such as depression and anxiety (MBSR, MBCT), addicts (MBRP), eating disorders (MBEAT), pain and chronic illness (MBPM). With this, Mindfulness has gained great popularity for a healthy population [6].

Thus, many studies have emphasized the structures and neurophysiology that Mindfulness practice promotes [7-9]. Based on these results, new protocols have been studied through body movement. The basis is that the body becomes an excellent anchor for maintaining attention, inasmuch attentional flexibility develops, the attentional process becomes faster and more natural [10]. An important factor in this finding is the main point that Mindfulness neurophysiology integrates different areas at different times of practice [11]. In the case of focused attention practice, attentional neural networks are present until distraction occurs, where more medial networks (default mode network) become more prominent $[12,13]$. As this distraction comes out, a deeper network (salience network) begins to activate so you can then decide to return to the focused object $[14,15]$.

This neural flow became known as the neurocognitive model $[11,14]$ that develops (neuroplasticity) throughout the training (hours/year of practice) [16].

Therefore, Mindfulness is no longer understood only as a practice of meditation or protocol but becomes a cognitive skill or mental training that develops as these neural networks adjust [17]. Then, body awareness is the starting point in the development of Mindfulness due to sensory experience improves the focus [18]. Thus, studies based on martial arts have been studied using Mindfulness neurophysiology as a major aim $[19,20]$. Studies using the Tai Chi Chuan [21] and Chi Kung [22] Mindfulness attitude had a great impact on research, showing important neurophysiological changes that corroborated with current findings that posterior areas (parietal cortex) of the cortex play a key role in practitioners' development [23-25]. Conscious movements have become a practical way of training Mindfulness, while martial arts have been an important way of maintaining these practices, considering the synchronization of movement with the Mindfulness attitudes described above.

Karate studies, for example, have gained prominence as training develops cognitive aspects such as attention, working memory, decision making $[17,26,27]$. The repetition of the movement seems to be an important way to manage the cognitive skill in this learning process, which is associated with a subtle recognition of the movement [26] with lower activity of the brainpower [28,29] and improved body balance [30]. Moreover, studies have shown improvement in cognitive processing speed in older practitioners [31] which related to neural efficiency [28,32]. More studies are necessary to explore the efficacy of martial arts training in the cognitive aspects, whereas Mindfulness has shown an important factor to link the physical activity into cognitive abilities.

\section{References}

1. Ussher M, Spatz A, Copland C, Nicolaou A, Cargill A, et al. (2014) Immediate Effects of a Brief Mindfulness-Based Body Scan on Patients with Chronic Pain. J Behav Med 37(1): 127-134. 
2. Chiesa A, Calati R, Serretti A (2011) Does Mindfulness Training Improve Cognitive Abilities? A Systematic Review of Neuropsychological Findings. Clin Psychol Rev 31(3): 449-464.

3. Galante J, Iribarren SJ, Pearce PF (2012) Effects of Mindfulness-Based Cognitive Therapy on Mental Disorders: A Systematic Review and MetaAnalysis of Randomised Controlled Trials. J Res Nurs 18(2): 133-155.

4. Kristeller JL, Hallett CB (1999) An Exploratory Study of a Meditationbased Intervention for Binge Eating Disorder. J Health Psychol 4(3): 357363.

5. Brown CA, Jones AKP (2012) Psychobiological Correlates of Improved Mental Health in Patients With Musculoskeletal Pain After a Mindfulnessbased Pain Management Program. Clin J Pain 29(3): 233-244.

6. Dickenson J, Berkman ET, Arch J, Lieberman MD (2012) Neural correlates of focused attention during a brief mindfulness induction. Soc Cogn Affect Neurosci 8(1): 40-47.

7. Fox KC, Nijeboer S, Dixon ML, Floman JL, Ellamil M, et al. (2014) Is Meditation Associated with Altered Brain Structure? A Systematic Review and Meta-Analysis of Morphometric Neuroimaging in Meditation Practitioners. Neurosci Biobehav Rev 43: 48-73.

8. Lomas T, Ivtzan I, Fu CHY(2015)A Systematic Review of the Neurophysiology of Mindfulness on EEG Oscillations. Neurosci Biobehav Rev 57: 401-410.

9. Chiesa A, Serretti A (2010) A Systematic Review Of Neurobiological and Clinical Features of Mindfulness Meditations. Psychol Med 40(8): 1239 1252.

10. Kerr CE, Jones SR, Wan Q, Pritchett DL, Wasserman RH et al. (2011) Effects of Mindfulness Meditation Training on Anticipatory Alpha Modulation in Primary Somatosensory Cortex 85(3-4): 96-103.

11. Malinowski P (2013) Neural mechanisms of attentional control in mindfulness meditation. Front Neurosci 7: 8.

12. Taylor V a, Daneault V, Grant J, Scavone G, Breton E, et al. (2013) Impact of meditation training on the default mode network during a restful state. Soc Cogn Affect Neurosci 8(1): 4-14.

13. Berkovich Ohana A, Glicksohn J, Goldstein A (2014) Studying the default mode and its mindfulness-induced changes using EEG functional connectivity. Soc Cogn Affect Neurosci 9(10): 1616-1624.

14. Hasenkamp W, Wilson-Mendenhall CD, Duncan E, Barsalou LW (2012) Mind wandering and attention during focused meditation: a finegrained temporal analysis of fluctuating cognitive states. Neuroimage 59(1): 750-760.

15. Kaunhoven RJ, Dorjee D (2017) How does mindfulness modulate selfregulation in pre-adolescent children? An integrative neurocognitive review. Neurosci Biobehav Rev 74(A): 163-184.

16. Russell T, Tatton-Ramos T (2014) Body in Mind Training: Mindful Movement for the Clinical Setting. Neuro-Disability Psychother 2(1/2): 108-136.

17. Johnstone A, Marí-Beffa P (2018) The Effects of Martial Arts Training on Attentional Networks in Typical Adults. Front Psychol 8: 9:80.
18. Kerr CE, Sacchet MD, Lazar SW, Moore CI, Jones SR (2013) Mindfulness starts with the body: somatosensory attention and top-down modulation of cortical alpha rhythms in mindfulness meditation. Front Hum Neurosci 7: 12.

19. Russell TA, Arcuri SM (2015) A Neurophysiological and Neuropsychological Consideration of Mindful Movement: Clinical and Research Implications. Front Hum Neurosci 9: 282.

20. Tang YY, Hölzel BK, Posner MI (2015) The neuroscience of mindfulness meditation. Nat Rev Neurosci 16(4):213-225

21. Zou L, Yeung A, Quan X, Boyden S, Wang H (2018) A Systematic Review and Meta-Analysis of Mindfulness-Based (Baduanjin) Exercise for Alleviating Musculoskeletal Pain and Improving Sleep Quality in People with Chronic Diseases. Int J Environ Res Public Health 15(2): 206.

22. Langhorst J, Häuser W, Bernardy K, Lucius H, Settan M, et al. (2012) [Complementary and alternative therapies for fibromyalgia syndrome. Systematic review, meta-analysis and guideline]. Schmerz 26(3): 311317.

23. Tang YY, Posner MI (2009) Attention training and attention state training. Trends Cogn Sci 13(5): 222-227.

24. Tang YY, Hölzel BK, Posner MI (2015) Traits and states in mindfulness meditation. Nat Rev Neurosci 17(1): 59.

25. Posner MI, Rothbart MK, Tang Y (2015) Enhancing attention through training. Curr Opin Behav sci 4: 1-5.

26. Babiloni C, Marzano N, Infarinato F, Iacoboni M, Rizza G, et al. (2010) "Neural efficiency" of experts' brain during judgment of actions: a highresolution EEG study in elite and amateur karate athletes. Behav Brain Res 207(2): 466-475.

27. Babiloni C, Vecchio F, Cappa S, Pasqualetti P, Rossi S et al. (2006) Functional frontoparietal connectivity during encoding and retrieval processes follows HERA model. A high-resolution study. Brain Res Bull 68(4): 203-212.

28. Babiloni C, Marzano N, Iacoboni M, Infarinato F, Aschieri P et al. (2010) Resting state cortical rhythms in athletes: a high-resolution EEG study. Brain Res Bull 81(1): 149-156.

29. Del Percio C, Infarinato F, Marzano N, Iacoboni M, Aschieri P, et al. (2011) Reactivity of alpha rhythms to eyes opening is lower in athletes than non-athletes: A high-resolution EEG study. Int J Psychophysiol 82(3): 240-247.

30. Del Percio C, Brancucci A, Bergami F, Marzano N, Fiore A, et al. (2007) Cortical alpha rhythms are correlated with body sway during quiet open eyes standing in athletes: A high-resolution EEG study. Neuroimage 36(3): 822-829.

31. Jansen P, Dahmen Zimmer K, Kudielka BM, Schulz A (2019) Effects of Karate Training Versus Mindfulness Training on Emotional Well-Being and Cognitive Performance in Later Life.

32. Duru AD, Assem M (2018) Investigating neural efficiency of elite karate athletes during a mental arithmetic task using EEG. Cogn Neurodyn 12(1): 95-102. 
(C) (1) This work is licensed under Creative

To Submit Your Article Click Here: Submit Article

DOI: $10.32474 /$ СTBM.2019.01.000111

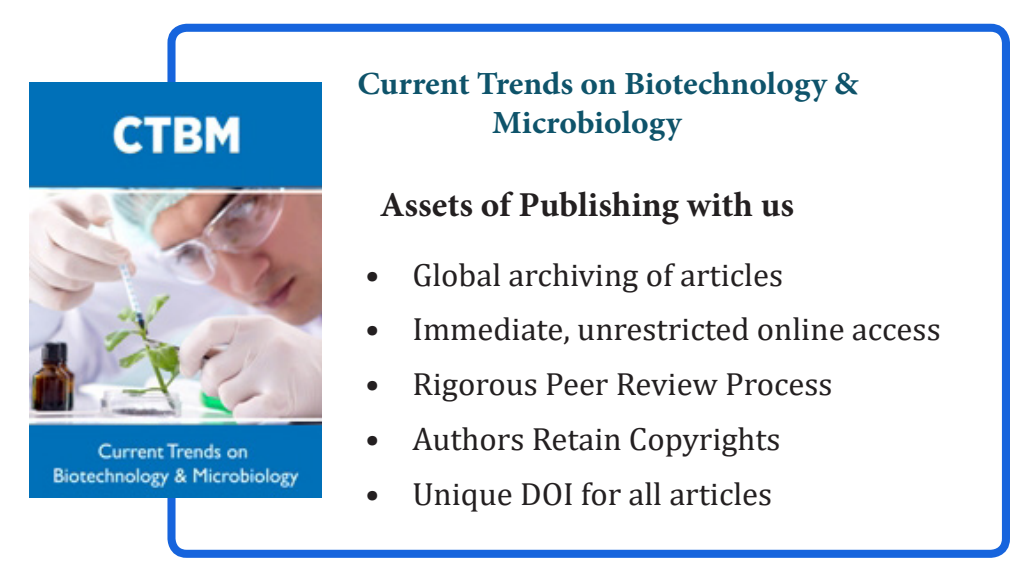

